\title{
Comparative Analysis of Transcriptional Gene Regulation Indicates Similar Physiologic Response in Mouse Tissues at Low Absorbed Doses from Intravenously Administered ${ }^{211}$ At
}

\author{
Britta Langen ${ }^{1,2}$, Nils Rudqvist ${ }^{1}$, Toshima Z. Parris ${ }^{3}$, Emil Schüler ${ }^{1}$, Khalil Helou $^{3}$, and Eva Forssell-Aronsson ${ }^{1}$ \\ ${ }^{I}$ Department of Radiation Physics, Institute of Clinical Sciences, Sahlgrenska Cancer Center, Sahlgrenska Academy, University of \\ Gothenburg, Sahlgrenska University Hospital, Gothenburg, Sweden; ${ }^{2}$ Department of Applied Physics, Chalmers University of \\ Technology, Gothenburg, Sweden; and ${ }^{3}$ Department of Oncology, Institute of Clinical Sciences, Sahlgrenska Cancer Center, \\ Sahlgrenska Academy, University of Gothenburg, Sahlgrenska University Hospital, Gothenburg, Sweden
}

\begin{abstract}
${ }^{211} \mathrm{At}$ is a promising therapeutic radionuclide because of the nearly optimal biological effectiveness of emitted $\alpha$-particles. Unbound ${ }^{211}$ At accumulates in the thyroid gland and in other vital normal tissues. However, few studies have been performed that assess the ${ }^{211} \mathrm{At}$-induced normal-tissue damage in vivo. Knowledge about the extent and quality of resulting responses in various organs offers a new venue for reducing risks and side effects and increasing the overall well-being of the patient during and after therapy. Methods: Female BALB/c nude mice were injected intravenously with $0.064-42 \mathrm{kBq}$ of ${ }^{211} \mathrm{At}$ or mock-treated, and the kidneys, liver, lungs, and spleen were excised $24 \mathrm{~h}$ after injection. A transcriptional gene expression analysis was performed in triplicate using RNA microarray technology. Biological processes associated with regulated transcripts were grouped into 8 main categories with 31 subcategories according to gene ontology terms for comparison of regulatory profiles. Results: A substantial decrease in the total number of regulated transcripts was observed between 0.64 and $1.8 \mathrm{kBq}$ of ${ }^{211}$ At for all investigated tissues. Few genes were differentially regulated in each tissue at all absorbed doses. In all tissues, most of these genes showed a nonmonotonous dependence on absorbed dose. However, the direction of regulation generally remained uniform for a given gene. Few known radiation-associated genes were regulated on the transcriptional level, and their expression profile generally appeared to be dose-independent and tissue-specific. The regulatory profiles of categorized biological processes were tissue-specific and reflected the shift in regulatory intensity between 0.64 and $1.8 \mathrm{kBq}$ of ${ }^{211} \mathrm{At}$. The profiles revealed strongly regulated and nonregulated subcategories. Conclusion: The strong regulatory change observed between 0.64 and $1.8 \mathrm{kBq}$ is hypothesized to result not only from low-dose effects in each tissue but also from physiologic responses to ionizing radiation-induced damage to, for example, the ${ }^{211} \mathrm{At}-$ accumulating thyroid gland. The presented results demonstrate the complexity of responses to radionuclides in vivo and highlight the need for further research to also consider physiology in ionizing radiation-induced responses.
\end{abstract}

Received Sep. 18, 2012; revision accepted Dec. 27, 2012.

For correspondence or reprints contact: Britta Langen, Department of Radiation Physics, University of Gothenburg, Sahlgrenska University Hospital, SE-413 45 Gothenburg, Sweden.

E-mail: britta.langen@gu.se

Published online May 8, 2013.

COPYRIGHT @ 2013 by the Society of Nuclear Medicine and Molecular Imaging, Inc.
Key Words: ${ }^{211} \mathrm{At}$; normal tissue response; transcriptional gene regulation; biological processes; radionuclide therapy

J Nucl Med 2013; 54:990-998

DOI: 10.2967/jnumed.112.114462

$\mathbf{T}$ he synthetic radiohalogen ${ }^{211} \mathrm{At}$ is a promising radionuclide for internal radiation therapy, because the linear energy transfer value of emitted $\alpha$-particles is nearly optimal for inducing DNA double-strand breaks $(1,2) .{ }^{211}$ Atlabeled tumor-seeking agents have been investigated for treatment of various tumor types, showing good prospects (3-6). However, during metabolism or degradation of radiolabeled compounds, ${ }^{211}$ At might be released and accumulated in the thyroid gland via mechanisms similar-if to a lesser degree- to radioiodine (7-11). Unbound ${ }^{211}$ At has been proposed for therapy of anaplastic thyroid carcinoma, yet the high uptake or retention in other normal tissues-for example, the kidneys, liver, lungs, and spleen-would limit successful therapy (12). The thyroid gland is required primarily for the hormonal regulation of metabolic processes in the body. Consequently, ionizing radiation-induced damage to the thyroid would affect the normal metabolic function of organs and eventually compromise physiologic integrity. At the same time, these organs could be affected by low absorbed doses from ${ }^{211}$ At as well. However, to the best of our knowledge, no study has been performed in a mammalian model system investigating normal tissue damage from low-dose exposure to ${ }^{211}$ At while also considering effects from a predominantly radiation-affected organ on the physiologic level. The microarray assay facilitates a genome-wide study on transcriptional gene regulation in response to stimulus without the risk of excluding regulatory pathways by focusing on a designated set of genes for detecting a presupposed response $(13,14)$. Furthermore, most studies focusing on radiation-induced transcriptional changes for the establishment of biomarkers have been performed in vitro $(15,16)$. This gap in basic knowledge 
and the increasing interest in ${ }^{211} \mathrm{At}$ for radionuclide therapy demand further research in the field of normal-tissue damage, which offers a new venue for optimizing treatment protocols and improving acute and long-term risk assessment. Ultimately, this continued research would increase treatment success rates and the overall well-being of the patient during and after therapy.

A previous study has demonstrated distinct transcriptional gene expression profiles in the thyroid of female $\mathrm{BALB} / \mathrm{c}$ nude mice at $24 \mathrm{~h}$ after the intravenous administration of ${ }^{211}$ At and showed dose-dependent gene regulation responses (17). Gene regulation responses in vivo are a complex topic, because the responses are affected by signals on the organizational level of cells, tissues, organs, and the organism as a whole. In this regard, ${ }^{211}$ At-induced radiation effects - which predominantly affect the thyroid—are also not expected to remain silent on the organismic level. The orchestrated hormonal regulation would be changed by radiation-induced effects on the thyroid and consequently affect other organs on the physiologic level as well. Moreover, intercellular and systemic signaling in vivo as a response to radiation also need to be considered. Cellular and molecular signaling have been identified as the basic mechanisms of radiation responses, thus also implying diverse local and systemic regulation mechanisms downstream of initial radiation-induced damage and repair (18-20).

The aim of this study was to elucidate the impact of $\alpha$-particle exposure from the intravenous administration of ${ }^{211}$ At on transcriptional gene regulation in normal tissues of the kidney cortex and medulla, liver, lungs, and spleen. The low- to medium-activity range of injected ${ }^{211} \mathrm{At}$ and resulting low absorbed doses were chosen to meet the relevance for both radiation protection and clinical use.

\section{MATERIALS AND METHODS}

\section{Radionuclides and Radioactivity Measurements}

The ${ }^{209} \mathrm{Bi}(\alpha, 2 \mathrm{n})^{211} \mathrm{At}$ reaction was used for the production of ${ }^{211} \mathrm{At}$ at the Cyclotron and PET Unit at Rigshospitalet in Copenhagen, Denmark. Free ${ }^{211}$ At was prepared as described by Lindegren et al. (21). Before and after injection, the activity in ${ }^{211}$ At stock solutions and syringes was measured using a $\gamma$-counter (1480 Wizard 3"; Wallac Oy).

\section{Calculation of Absorbed Dose}

Organ-specific mean absorbed doses $\left(\bar{D}_{\text {organ }}\right)$ were calculated according to the MIRD formalism (22):

$$
\bar{D}_{\text {organ }}=\frac{\tilde{A}_{\text {organ }} \times \sum_{i} n_{i} E_{i} \Phi_{i}}{m_{\text {organ }}},
$$

where $\tilde{A_{\text {organ }}}$ is the cumulated activity of ${ }^{211} \mathrm{At}$ in the respective organ (or tissue) at a given time, $n_{i}$ is the radiation yield with energy $E_{i}$ and absorbed fraction $\Phi_{i}$ in the target organ (or tissue), and $m_{\text {organ }}$ is the mass of the respective organ. Calculations of organ-specific cumulated activities were based on biodistribution data, giving the percentage of injected activity for various organs over a time range from 0 to $24 \mathrm{~h}$ (23). Activity distributions were assumed to be homogeneous for all organs. The derivative of the cumulated activity between the given time points $0,1,2,4,6$, and $24 \mathrm{~h}$ was formed according to the trapezoidal rule. Only the contributions from the emitted $\alpha$-particles from ${ }^{211} \mathrm{At}$ and its daughter ${ }^{211} \mathrm{Po}$ were considered. Because of the short mean range of ${ }^{211} \mathrm{At}-$ emitted $\alpha$-particles $(\sim 65 \mu \mathrm{m})$, the absorbed fraction was set to 1 for all investigated organs (24).

\section{Animal Experiments}

Twenty-one 6-mo-old female BALB/c nude mice (Charles River) were divided into 5 experimental groups and 1 control group. Solutions of ${ }^{211} \mathrm{At}$ with $0.064,0.64,1.8,14$, and $42 \mathrm{kBq}$ (in phosphate-buffered saline at $\mathrm{pH} 7$ ) were injected into the tail vein. The control group was mock-treated with an empty syringe. Animals were anesthetized with sodium pentobarbital and killed $24 \mathrm{~h}$ after injection via cardiac puncture. The kidneys, liver, lungs, and spleen were excised and flash-frozen in liquid nitrogen before storage at $-80^{\circ} \mathrm{C}$ until analysis. The procedures were conducted as approved by the Ethical Committee on Animal Experiments in Gothenburg, Sweden.

\section{Gene Expression Analysis}

Several milligrams of sample material were dissected on dry ice from the stored organs; kidney samples were divided into cortical and medullary tissues and treated separately. Samples from mice in the same group were pooled. Total RNA was extracted using the RNeasy Lipid Tissue Mini Kit (Qiagen) according to the manufacturer's instructions. The RNA integrity number was determined with the RNA 6000 Nano LabChip Kit (Agilent Technologies) using the 2100 Bioanalyzer (Agilent Technologies) according to the standard protocol. Samples with an RNA integrity number below 6.0 were not used in subsequent analyses.

The RNA samples were processed in triplicate at the Swegene Center for Integrative Biology Genomics DNA Microarray Resource Center (SCIBLU). Hybridizations were performed on MouseRef-8 Whole-Genome Expression BeadChips (Illumina). The BeadArray Reader scanner (Illumina) was used for image acquisition, and BeadScan 3.5.31.17122 image analysis software (Illumina) was used for the quantification of raw signal intensities. The web-based BioArray Software Environment system provided by SCIBLU was used for data preprocessing and quantile normalization of raw signal intensities. Further data processing was performed with Nexus Expression 2.0 (BioDiscovery) as described elsewhere (25).

The false-discovery rate was controlled with an adjusted $P$ value cutoff of less than 0.01 for differentially expressed transcripts with a threshold of a 1.5 -fold change or more according to the Benjamini-Hochberg method described elsewhere (26). The $P$ value cutoff for the identification of enriched gene ontology (GO) terms associated with a gene set was set to a $P$ value of less than 0.05 . Obtained data were further analyzed via web-based GO term searches in the GO database (http://www.geneontology. org) (27). Biological processes associated with regulated transcripts were categorized using GO terms and ancestor charts to establish regulatory profiles that facilitated comparison on 2 dimensions - that is, between absorbed doses of a tissue and between tissues at a given exposure. Eight main categories with 31 subcategories were distinguished that account for higher-level cellular functions. The gene expression data presented in this study have been deposited in the Gene Expression Omnibus (GEO accession GSE40806) of the National Center for Biotechnology Information. 
TABLE 1

Tissue-Specific Absorbed Doses Over 24 Hours (mGy)

\begin{tabular}{llccrr}
\hline & \multicolumn{5}{c}{211 At activity $(\mathrm{kBq})$} \\
\cline { 2 - 6 } Mouse tissue & 0.064 & 0.64 & 1.8 & 14 & \multicolumn{1}{c}{42} \\
\hline Kidney cortex & 0.24 & 2.4 & 6.9 & 52 & 160 \\
Kidney medulla & 0.24 & 2.4 & 6.9 & 52 & 160 \\
Liver & 0.2 & 2 & 5.5 & 44 & 130 \\
Lungs & 1.6 & 16 & 45 & 350 & 1,000 \\
Spleen & 0.45 & 4.5 & 13 & 98 & 300
\end{tabular}

Calculation of tissue-specific absorbed doses at $24 \mathrm{~h}$ after intravenous administration of ${ }^{211} \mathrm{At}$ based on MIRD formalism (22).

\section{RESULTS}

\section{Organ-Specific Absorbed Doses}

Intravenously injected solutions of ${ }^{211} \mathrm{At}(0.064-42 \mathrm{kBq})$ resulted in mean absorbed doses in the very-low- to lowdose range in the mouse kidneys, liver, lungs, and spleen (Table 1). The liver showed the lowest absorbed doses, ranging between 0.20 and $130 \mathrm{mGy}$. The kidneys showed approximately 1.2-fold-higher values, whereas the spleen was exposed to approximately 2.3-fold-higher absorbed doses than the liver. The lungs received distinctively higher absorbed doses, with 1,000 mGy at the highest injected activity, which was approximately 3.3-fold higher than for the spleen, 6.3-fold higher than for the kidneys, and 7.7fold higher than for the liver.

\section{Similarities in Transcriptional Gene Regulation}

Global gene expression profiles were assessed by the total number of significantly regulated transcripts (adjusted $P$ value $<0.01)$ at a given absorbed dose, compared with controls. At $24 \mathrm{~h}$ after injection, clear regulatory effects were observed at all absorbed dose levels in all investigated tissues (Fig. 1). Furthermore, the tissues showed 2 common characteristics in the total transcriptional response. First, despite differences in organ-specific absorbed doses, a pronounced decrease in the extent of both up- and downregulated transcripts was seen for all tissues between 0.64 and $1.8 \mathrm{kBq}$ of ${ }^{211} \mathrm{At}$. Considering all tissues at respective activities, the number of upregulated transcripts decreased from 538-823 to 68-173, whereas the number of downregulated transcripts decreased from 139-479 to 18-251. Second, upregulation dominated over downregulation for all tissues and at all absorbed doses. Only the kidney medulla presented 2 exceptions from these common characteristics. First, at $1.8 \mathrm{kBq}$ and higher activities, downregulation was more pronounced than upregulation, and second, from 0.64 to $1.8 \mathrm{kBq}$, the number of downregulated transcripts increased from 188 to 251 transcripts.

No gene was differentially expressed in all organs across all exposures at $24 \mathrm{~h}$ after ${ }^{211}$ At injection. One gene, Angptl4 (angiopoietinlike protein 4), nearly attained ubiquitous expression, being regulated in 23 of the 25 tuples (5 tissues across 5 activities). Regulation of Angptl4 was not observed in the liver at 0.064 and $1.8 \mathrm{kBq}$. For the kidney cortex and medulla, lungs, and spleen, between 3 and 5 genes were differentially expressed at all absorbed doses (Fig. 2). The liver exhibited a somewhat higher number of regulated genes, dominated by downregulation $(9 / 15$ genes) (Fig. 3). Most genes showed a nonmonotonous dependence on absorbed dose for all tissues. However, the direction of regulation generally remained uniform.

\section{Regulation of Radiation-Associated Genes}

The differential expression of 56 radiation-associated genes proposed by Snyder and Morgan (15) and Chaudhry (16) showed low response levels at $24 \mathrm{~h}$ after ${ }^{211}$ At injection. Only 19 genes showed significant regulation at least in 1 tissue (Fig. 4), whereas most genes (37/56) showed no significant regulation in any tissue at any exposure. In
FIGURE 1. Comparison of total number (no.) of significantly up- and downregulated transcripts (positive and negative numbers, respectively) in kidney cortex and medulla, liver, lungs, and spleen at $24 \mathrm{~h}$ after intravenous administration of $0.064-42 \mathrm{kBq}$ of ${ }^{211}$ At.

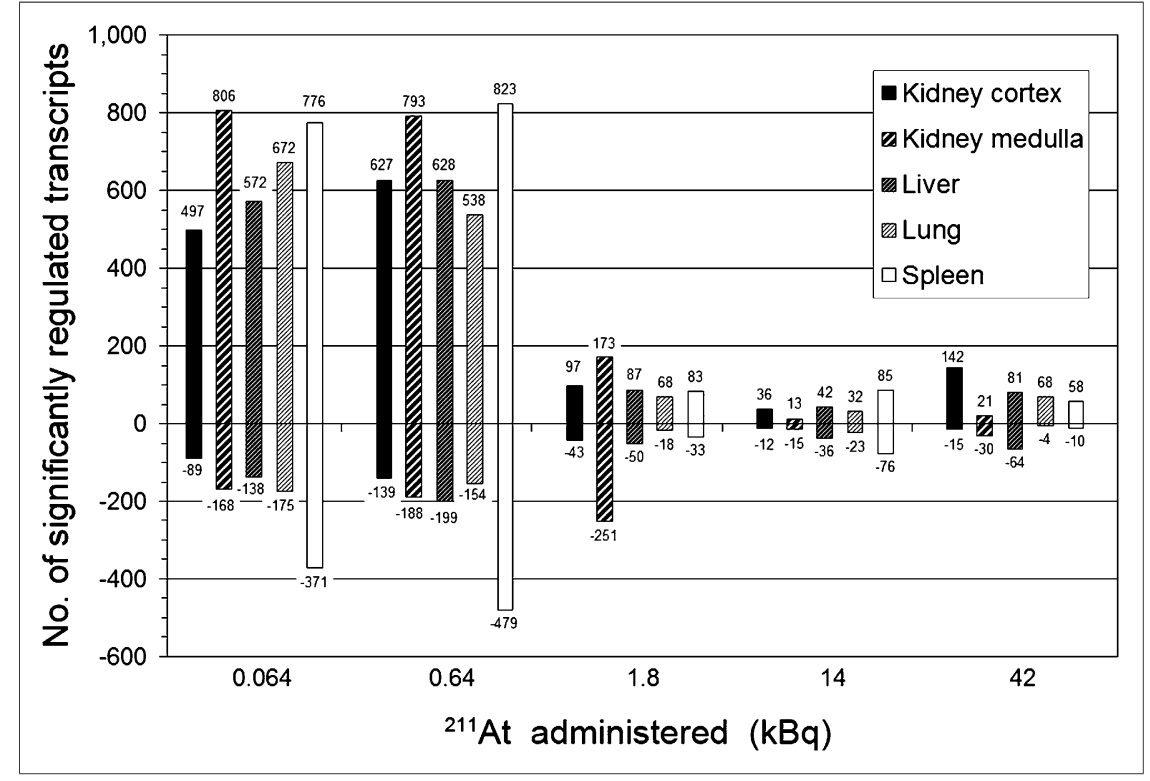




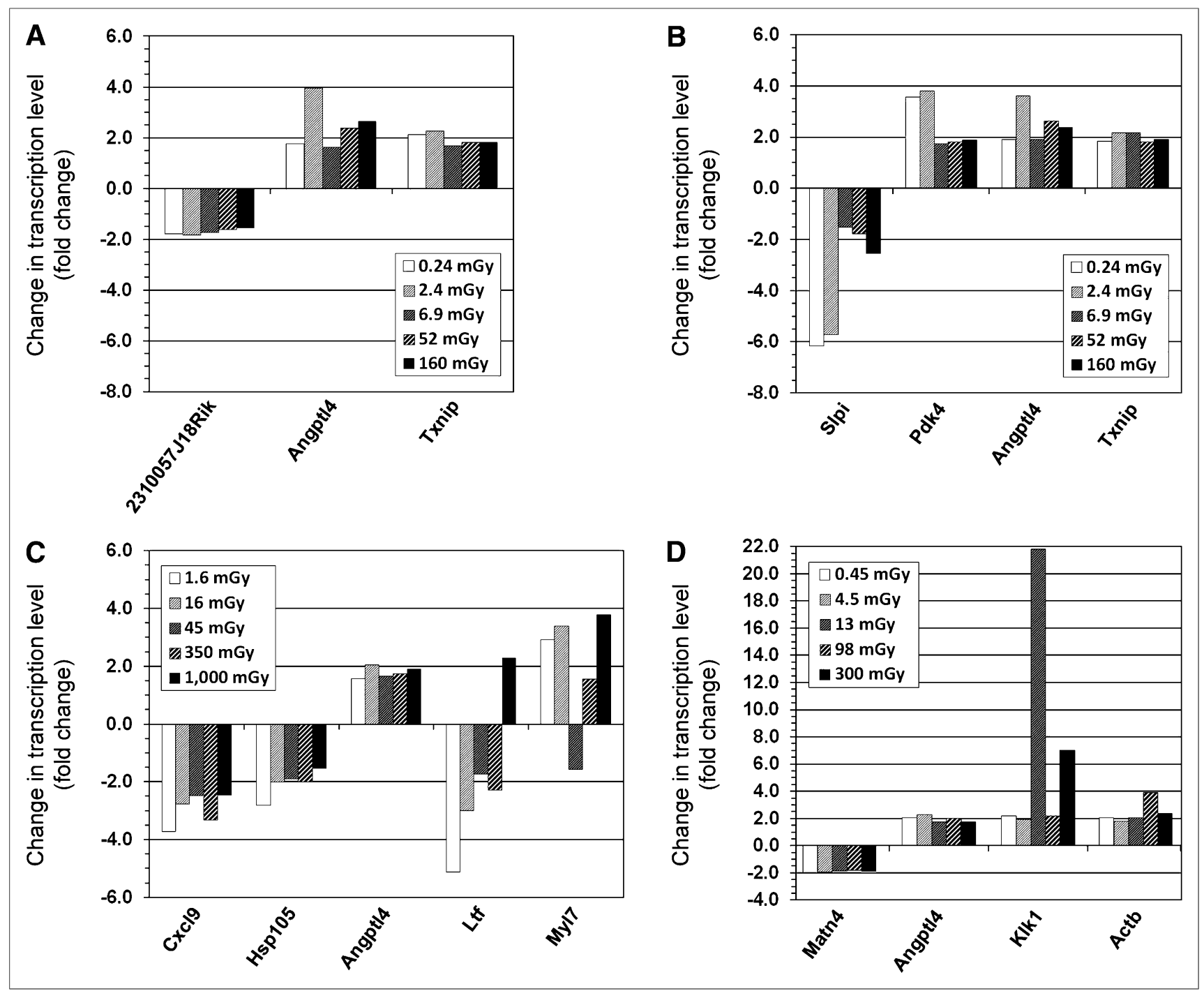

FIGURE 2. Differentially regulated genes on transcription level in kidney cortex (A), kidney medulla (B), lungs (C), and spleen (D) at all absorbed doses from ${ }^{211} \mathrm{At}$ at $24 \mathrm{~h}$ after intravenous injection. Note different scales on $y$-axes.

general, the type and number of regulated genes within the radiation-associated signature and their regulation incidences-that is, regulation of a given gene at one or more absorbed doses-appeared specific for each tissue. The strength of regulation was generally the same for upand downregulation in all tissues. Downregulation ranged from a -1.5 -fold change to a -2.8 -fold change. Upregulation ranged from a 1.5 -fold change to a 2.3 -fold change. The only exception was regulation of Amy2 (encoding for the pancreatic $\alpha$-amylase 2a5) in the spleen, where upregulation for absorbed doses of 13, 98, and $300 \mathrm{mGy}$ resulted in 133-, 19-, and 87-fold changes, respectively. Pcna (proliferating cell nuclear antigen) was the only radiation-associated gene regulated for at least 1 exposure in each tissue: the spleen showed consistent downregulation at the 3 highest injected activities, whereas all other tissues showed 1 or 2 incidences of upregulation only at the 2 lowest injected activities. In all cases, the regulation of Pcna was at a similarly low level.

\section{Affected Biological Processes}

The transcriptional regulation of biological processes displayed diverse profiles for each tissue when categorized after higher-level cellular function (Fig. 5). A clear shift in the transcriptional response was observed between 0.64 and $1.8 \mathrm{kBq}$ of ${ }^{211} \mathrm{At}$, with a pronounced decrease in the number of regulated transcripts. This shift is further referred to as threshold behavior in terms of 2 different phases of regulatory intensity. The kidney cortex and medulla, liver, and lungs showed some regulation incidences for maintenance of DNA integrity, with no observable regulation of DNA damage and repair processes. The regulation profile in the spleen exhibited threshold behavior, with a clear decrease between 0.64 and $1.8 \mathrm{kBq}$ (4.5 and $13 \mathrm{mGy}$, respectively) 


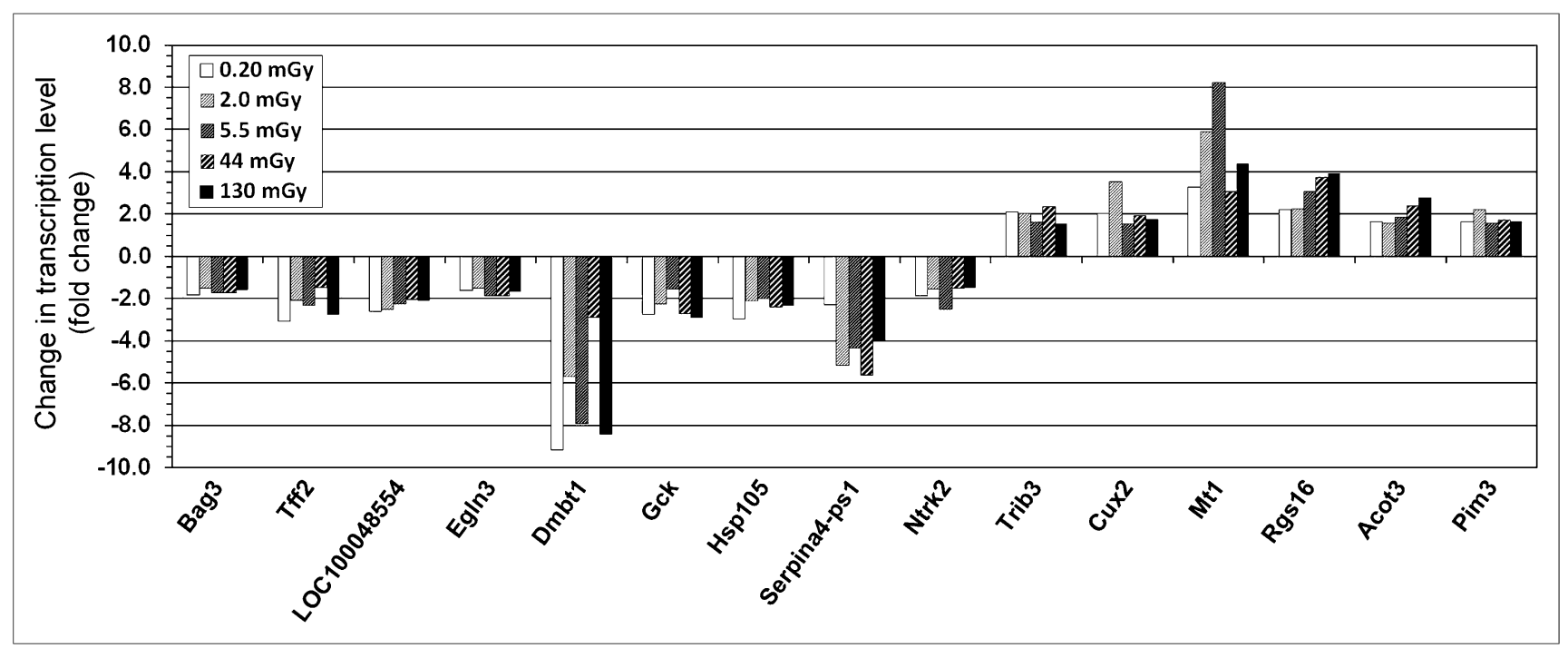

FIGURE 3. Differentially regulated genes on transcription level in liver at all absorbed doses from ${ }^{211} \mathrm{At}$ at $24 \mathrm{~h}$ after intravenous injection. Note scaling of $y$-axis.

for maintenance of DNA integrity, also showing a clear regulatory response for DNA damage and repair processes. All tissues showed threshold behavior, with a decrease in response intensity between 0.64 and $1.8 \mathrm{kBq}$ for the regulation of gene expression integrity and cellular integrity. However, the extent of this decrease varied and showed tissue-specific divergent patterns on the subcategory level. Threshold behav- ior was also observed for the regulation of cell cycle and differentiation in the kidney medulla, liver, and spleen, whereas the kidney cortex and lungs did not show this characteristic. Cell communication was regulated on a generally low level, without observable threshold behavior in all tissues except for the spleen, which showed a strong regulation response in signal transduction below the observed threshold.

\begin{tabular}{|c|c|c|c|c|c|c|c|c|c|c|c|c|c|c|c|c|c|c|c|c|}
\hline & $\begin{array}{l}\text { Gene } \\
\text { symbol: }\end{array}$ & 彥 & กิ & : & క్ర & ¿্ّ & : & 旁 & 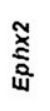 & ¿ & 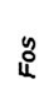 & 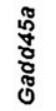 & 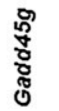 & 花 & ิิ & $\stackrel{\Xi}{\Xi}$ & $\stackrel{\infty}{\stackrel{\infty}{\leftrightarrows}}$ & 窨 & 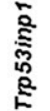 & : \\
\hline & $\begin{array}{l}\text { Absorbed dose } \\
\text { (mGy): }\end{array}$ & & & & & & & & & & Id ch & ge & & & & & & & & \\
\hline \multirow{5}{*}{$\begin{array}{l}\text { Kidney } \\
\text { cortex }\end{array}$} & 0.24 & & & & & & & & & & & & & & & & & & & \\
\hline & 2.4 & & & & & & & & & & & & 1.5 & & & 1.6 & & & & \\
\hline & 6.9 & & & & & & & & & & & & & & & & & & & \\
\hline & 52 & & & & & & & & & & & & & & & & & & & \\
\hline & 160 & & & & & & & & & & & & & & & & & 1.6 & & \\
\hline \multirow{5}{*}{$\begin{array}{l}\text { Kidney } \\
\text { medulla }\end{array}$} & 0.24 & 1.7 & & & -1.6 & & & & 1.8 & & & 1.5 & & 1.8 & & 1.7 & 1.5 & & & -1.5 \\
\hline & 2.4 & & & & & & & & 2.0 & & & & & & & 1.5 & 1.6 & & & -1.8 \\
\hline & 6.9 & & & & & & -1.8 & & & & 1.5 & & & & & & & & & \\
\hline & 52 & & & & & & & & & & & & & & & & & & & \\
\hline & 160 & & & & & & & & & & & & & & & & & & & \\
\hline \multirow{5}{*}{ Liver } & 0.20 & 2.0 & 2.2 & & -1.8 & & & & & & 1.6 & & & & & 1.7 & & & & \\
\hline & 2.0 & & & & & & & & & & & -1.5 & & & -1.6 & & & & & \\
\hline & 5.5 & & 1.7 & & & & & & & & & & & & & & & & & \\
\hline & 44 & & & & & & & & & & & & -2.1 & & & & & & & \\
\hline & 130 & & & & & & & & & & & & -2.4 & & & & & & & \\
\hline \multirow{5}{*}{ Lung } & 1.6 & & & & & & & & & & 2.2 & & & & & 1.6 & 1.6 & & 2.3 & -1.5 \\
\hline & 16 & & & & & & & & & & & & & & & 1.5 & & & 1.6 & \\
\hline & 45 & & & & & & & & & & & & & & & & & & & \\
\hline & 350 & & & & & & & & & & & & & & & & & & 1.5 & \\
\hline & 1000 & & & & & & & & & & & & & & & & & & & \\
\hline \multirow{5}{*}{ Spleen } & 0.45 & & & & & -1.8 & & & & -2.3 & & & & & & & & & & -1.7 \\
\hline & 4.5 & & & -2.1 & & -2.1 & & 1.5 & & -2.8 & & -2.2 & & & & & & & & -1.8 \\
\hline & 13 & & 133 & & & & & & & & & & & & & -1.6 & & & & \\
\hline & 98 & & 19 & & & & & & & & & & & & & -1.8 & & & & \\
\hline & 300 & & 87 & & & & & & & & & & & & & -1.5 & & & & \\
\hline
\end{tabular}

FIGURE 4. Transcriptional regulation of previously proposed radiation-associated genes $(15,16)$ at $24 \mathrm{~h}$ after intravenous administration of ${ }^{211}$ At. 


\begin{tabular}{|c|c|c|c|c|c|c|c|c|c|c|c|c|c|c|c|c|c|c|c|c|c|c|c|c|c|c|}
\hline \multirow{2}{*}{$\begin{array}{l}\text { No. (filtered } \\
\text { transcripts) }\end{array}$} & \multirow{2}{*}{$\begin{array}{r}\text { Mouse organ: } \\
\text { Absorbed dose ( } \mathrm{mGy} \text { : }\end{array}$} & \multicolumn{5}{|c|}{ Kidney cortex } & \multicolumn{5}{|c|}{ Kidney medulla } & \multicolumn{5}{|c|}{ Liver } & \multicolumn{5}{|c|}{ Lung } & \multicolumn{5}{|c|}{ Spleen } \\
\hline & & 0.24 & 2.4 & 6.9 & 52 & 160 & 0.24 & 2.4 & 6.9 & 52 & 160 & 0.20 & 2.0 & 5.5 & 44 & 130 & 1.6 & 16 & 453 & 350 & 1000 & $\overline{0.45}$ & 4.5 & 13 & 98 & 300 \\
\hline 109 & DNA integrity & 4 & 0 & 0 & 0 & 0 & 0 & 3 & 17 & 0 & 0 & 0 & 0 & 1 & 0 & 1 & 3 & 0 & 0 & 0 & 0 & 22 & 41 & 3 & 1 & 1 \\
\hline 30 & Damage and repair & 0 & 0 & 0 & 0 & 0 & 0 & 0 & 0 & 0 & 0 & 0 & 0 & 0 & 0 & 0 & 0 & 0 & 0 & 0 & 0 & 0 & 12 & 1 & 1 & 1 \\
\hline 79 & Chromatin organization & 4 & 0 & 0 & 0 & 0 & 0 & 3 & 17 & 0 & 0 & 0 & 0 & 1 & 0 & 1 & 3 & 0 & 0 & 0 & 0 & 22 & 29 & 2 & 0 & 0 \\
\hline 661 & Gene expression integrity & 4 & 112 & 0 & 0 & 0 & 21 & 21 & 5 & 0 & 2 & 115 & 145 & 0 & 0 & 0 & 30 & 15 & 2 & 0 & 1 & 181 & 194 & 0 & 0 & 0 \\
\hline 567 & Transcription & 2 & 103 & 0 & 0 & 0 & 0 & 0 & 0 & 0 & 1 & 96 & 118 & 0 & 0 & 0 & 0 & 0 & 0 & 0 & 0 & 161 & 171 & 0 & 0 & 0 \\
\hline 64 & RNAprocessing & 0 & 9 & 0 & 0 & 0 & 17 & 11 & 0 & 0 & 0 & 17 & 18 & 0 & 0 & 0 & 21 & 11 & 2 & 0 & 0 & 20 & 23 & 0 & 0 & 0 \\
\hline 30 & Translation & 2 & 0 & 0 & 0 & 0 & 4 & 10 & 5 & 0 & 1 & 2 & 9 & 0 & 0 & 0 & 9 & 4 & 0 & 0 & 1 & 0 & 0 & 0 & 0 & 0 \\
\hline 1054 & Cellular integrity & 75 & 143 & 11 & 8 & 50 & 110 & 128 & 29 & 3 & 6 & 35 & 19 & 6 & 9 & 6 & 67 & 73 & 5 & 8 & 16 & 45 & 47 & 2 & 0 & 0 \\
\hline 174 & Physico-chemical environment & 2 & 35 & 5 & 6 & 22 & 2 & 2 & 23 & 0 & 3 & 5 & 9 & 1 & 4 & 3 & 3 & 6 & 0 & 8 & 6 & 8 & 7 & 0 & 0 & 0 \\
\hline 139 & Cytoskeleton \& motility & 0 & 0 & 0 & 0 & 4 & 2 & 4 & 4 & 2 & 2 & 0 & 10 & 0 & 5 & 3 & 24 & 14 & 5 & 0 & 10 & 20 & 21 & 0 & 0 & 0 \\
\hline 152 & Extracellular matrix \& $\mathrm{CM}$ & 0 & 0 & 0 & 0 & 2 & 6 & 0 & 0 & 0 & 0 & 0 & 0 & 0 & 0 & 0 & 3 & 24 & 0 & 0 & 0 & 0 & 0 & 2 & 0 & 0 \\
\hline 589 & Supramolecular maintenance & 73 & 108 & 6 & 2 & 22 & 100 & 122 & 2 & 1 & 1 & 30 & 0 & 5 & 0 & 0 & 37 & 29 & 0 & 0 & 0 & 17 & 19 & 0 & 0 & 0 \\
\hline 510 & Cell cycle and differentiation & 9 & 8 & 9 & 0 & 3 & 24 & 18 & 2 & 2 & 9 & 33 & 22 & 6 & 5 & 4 & 7 & 11 & 6 & 2 & 4 & 126 & 190 & 7 & 25 & 2 \\
\hline 290 & Cell cycle regulation & 0 & 0 & 0 & 0 & 0 & 9 & 4 & 0 & 0 & 1 & 0 & 0 & 4 & 0 & 0 & 0 & 7 & 0 & 0 & 0 & 96 & 133 & 6 & 24 & 2 \\
\hline 50 & Differentiation \& aging & 7 & 4 & 7 & 0 & 0 & 7 & 6 & 2 & 2 & 4 & 6 & 4 & 2 & 2 & 1 & 5 & 4 & 1 & 1 & 2 & 6 & 4 & 1 & 1 & 0 \\
\hline 155 & Apoptotic cell death & 2 & 2 & 0 & 0 & 3 & 2 & 2 & 0 & 0 & 2 & 22 & 18 & 0 & 3 & 3 & 2 & 0 & 4 & 0 & 2 & 24 & 53 & 0 & 0 & 0 \\
\hline 13 & Cell death & 0 & 0 & 2 & 0 & 0 & 6 & 6 & 0 & 0 & 2 & 5 & 0 & 0 & 0 & 0 & 0 & 0 & 0 & 0 & 0 & 0 & 0 & 0 & 0 & 0 \\
\hline 2 & General & 0 & 2 & 0 & 0 & 0 & 0 & 0 & 0 & 0 & 0 & 0 & 0 & 0 & 0 & 0 & 0 & 0 & 1 & 1 & 0 & 0 & 0 & 0 & 0 & 0 \\
\hline 381 & Cell communication & 2 & 3 & 5 & 3 & 4 & 2 & 4 & 0 & 8 & 3 & 0 & 9 & 6 & 10 & 4 & 10 & 2 & 7 & 3 & 9 & 25 & 24 & 1 & 0 & 1 \\
\hline 16 & Intercellularsignaling & 2 & 2 & 3 & 0 & 0 & 2 & 4 & 0 & 0 & 0 & 0 & 0 & 0 & 1 & 0 & 2 & 0 & 5 & 0 & 5 & 4 & 0 & 0 & 0 & 0 \\
\hline 365 & Signal transduction & 0 & 1 & 2 & 3 & 4 & 0 & 0 & 0 & 8 & 3 & 0 & 9 & 6 & 9 & 4 & 8 & 2 & 2 & 3 & 4 & 21 & 24 & 1 & 0 & 1 \\
\hline 828 & Metabolism & 71 & 81 & 11 & 2 & 2 & 108 & 63 & 32 & 5 & 8 & 58 & 113 & 17 & 10 & 31 & 15 & 55 & 10 & 11 & 20 & 2 & 3 & 12 & 15 & 13 \\
\hline 62 & Proteins, amino acids & 0 & 0 & 1 & 0 & 0 & 3 & 0 & 3 & 0 & 0 & 0 & 12 & 0 & 4 & 3 & 6 & 4 & 0 & 2 & 0 & 0 & 0 & 1 & 6 & 1 \\
\hline 292 & Lipids, fatty acids & 9 & 48 & 5 & 2 & 2 & 7 & 8 & 12 & 3 & 8 & 14 & 43 & 14 & 2 & 19 & 0 & 8 & 5 & 4 & 5 & 0 & 3 & 11 & 9 & 12 \\
\hline 33 & Carbohydrates & 2 & 0 & 5 & 0 & 0 & 0 & 0 & 0 & 0 & 0 & 4 & 2 & 1 & 1 & 1 & 0 & 3 & 4 & 3 & 4 & 0 & 0 & 0 & 0 & 0 \\
\hline 18 & Signaling molecules & 0 & 2 & 0 & 0 & 0 & 5 & 7 & 0 & 0 & 0 & 0 & 0 & 1 & 1 & 0 & 0 & 2 & 1 & 0 & 2 & 2 & 0 & 0 & 0 & 0 \\
\hline 32 & Nucleic acid-related & 0 & 0 & 0 & 0 & 0 & 4 & 2 & 0 & 0 & 0 & 3 & 2 & 0 & 1 & 0 & 9 & 6 & 0 & 0 & 1 & 0 & 0 & 0 & 0 & 0 \\
\hline 41 & Other & 2 & 2 & 0 & 0 & 0 & 4 & 5 & 0 & 2 & 0 & 7 & 6 & 1 & 0 & 0 & 0 & 4 & 0 & 2 & 1 & 0 & 0 & 0 & 0 & 0 \\
\hline 350 & General & 58 & 29 & 0 & 0 & 0 & 85 & 41 & 17 & 0 & 0 & 30 & 48 & 0 & 1 & 8 & 0 & 28 & 0 & 0 & 7 & 0 & 0 & 0 & 0 & 0 \\
\hline 450 & Stress responses & 2 & 13 & 37 & 5 & 21 & 16 & 12 & 3 & 5 & 3 & 19 & 13 & 0 & 6 & 2 & 21 & 4 & 13 & 10 & 8 & 43 & 132 & 7 & 11 & 6 \\
\hline 24 & Oxidativestress response & 0 & 0 & 0 & 0 & 0 & 0 & 0 & 0 & 0 & 0 & 0 & 0 & 0 & 3 & 0 & 0 & 0 & 0 & 0 & 0 & 0 & 3 & 2 & 2 & 0 \\
\hline 41 & Inflammatory response & 0 & 0 & 0 & 0 & 0 & 0 & 0 & 0 & 3 & 0 & 0 & 0 & 0 & 0 & 0 & 0 & 0 & 1 & 0 & 0 & 0 & 17 & 0 & 6 & 3 \\
\hline 298 & Immune response & 2 & 2 & 36 & 4 & 21 & 5 & 2 & 0 & 0 & 2 & 9 & 6 & 0 & 2 & 1 & 8 & 0 & 6 & 8 & 4 & 43 & 98 & 3 & 2 & 2 \\
\hline 87 & Other & 0 & 11 & 1 & 1 & 0 & 11 & 10 & 3 & 2 & 1 & 10 & 7 & 0 & 1 & 1 & 13 & 4 & 6 & 2 & 4 & 0 & 14 & 2 & 1 & 1 \\
\hline 166 & Organismic regulation & 3 & 9 & 2 & 4 & 7 & 5 & 8 & 5 & 4 & 5 & 16 & 12 & 3 & 6 & 5 & 2 & 11 & 3 & 2 & 12 & 0 & 8 & 4 & 5 & 3 \\
\hline 8 & Behavior & 0 & 0 & 1 & 0 & 0 & 0 & 0 & 0 & 0 & 1 & 2 & 0 & 1 & 2 & 0 & 0 & 0 & 0 & 0 & 0 & 0 & 0 & 0 & 0 & 0 \\
\hline 97 & Ontogenesis & 0 & 4 & 0 & 1 & 5 & 0 & 3 & 3 & 2 & 1 & 8 & 9 & 0 & 2 & 1 & 2 & 7 & 3 & 1 & 3 & 0 & 8 & 1 & 1 & 0 \\
\hline 53 & Systemic regulation & 0 & 2 & 1 & 3 & 2 & 2 & 2 & 0 & 2 & 2 & 3 & 0 & 1 & 2 & 4 & 0 & 0 & 0 & 0 & 9 & 0 & 0 & 3 & 3 & 3 \\
\hline 8 & Reproduction & 3 & 3 & 0 & 0 & 0 & 3 & 3 & 2 & 0 & 1 & 3 & 3 & 1 & 0 & 0 & 0 & 4 & 0 & 1 & 0 & 0 & 0 & 0 & 1 & 0 \\
\hline
\end{tabular}

FIGURE 5. Regulation of biological processes categorized after higher-level cellular function: number (no.) of scored transcripts associated with genes regulating biological processes grouped in category or subcategory is shown. Value is number of regulated filtered transcripts (i.e., scored transcripts) at $24 \mathrm{~h}$ after intravenous administration of ${ }^{211} \mathrm{At}$. Total number of filtered transcripts grouped in category or subcategory is shown in left-hand column. Percentage of scored vs. filtered transcripts is described as very low $<3 \%$, low 3\%-9\%, medium $10 \%-29 \%$, high $30 \%-49 \%$, and very high $\geq 50 \%$ and highlighted as very light blue, light blue, blue, dark blue, very dark blue, respectively.

The regulation of metabolism showed the most diverse regulatory patterns: the threshold behavior was apparent only in the kidney cortex between 0.64 and $1.8 \mathrm{kBq}(2.4$ and $6.9 \mathrm{mGy}$, respectively). In contrast, liver and lungs displayed a peak in regulation of metabolism at $0.64 \mathrm{kBq}$ (2.0 and $16 \mathrm{mGy}$, respectively). The kidney medulla displayed a strong decrease in regulation of metabolism, with increased absorbed dose, whereas the spleen showed mainly a slight increase in regulation, with increased absorbed dose remaining at the same level at the highest absorbed dose. The regulation of stress responses was at a very-low to low level in all tissues except in the spleen at $0.64 \mathrm{kBq}(4.5 \mathrm{mGy})$ with 132 of $450(29 \%)$ transcripts, respectively. Notably, oxidative stress responses were not regulated at any absorbed dose in the kidney tissues and lungs, whereas regulation of inflammatory responses was not detected in the kidney cortex and liver. The regulation of immune responses in the spleen depicted threshold behavior, with medium to high regulation at the 2 lowest exposures (43/298 [14\%] and 98/298 [33\%], respectively). Biological processes involved in organismic regulation showed very-low to low regulation on the categoric level in all tissues. A complete list of categorized biological processes is provided in Supplemental Tables 1-5 (supplemental materials are available online only at http://jnm.snm journals.org).

\section{DISCUSSION}

The radiohalogen ${ }^{211} \mathrm{At}$ has a half-life of $7.2 \mathrm{~h}$ and decays via $\alpha$-emission and electron capture to ${ }^{207} \mathrm{Bi}$ and ${ }^{211}$ Po. The average energy of $\alpha$-particles-also considering $\alpha$-particle emission from ${ }^{211}$ Po due to its short half-life of approximately $0.5 \mathrm{~s}$-is $6.9 \mathrm{MeV}$. With a mean average range of approximately $65 \mu \mathrm{m}$ in tissue (24), it can be estimated that an emitted $\alpha$-particle traverses between 1 and 9 cell layers. For the thyroid, microdosimetric dose distributions on intravenous administration of ${ }^{211}$ At have been calculated and discussed in this context, stating that 
a mean absorbed dose of 1.2 Gy would correspond to a statistical mean value of $1 \alpha$-particle track per thyroid cell (28). Accordingly, absorbed doses of 50 and $500 \mathrm{mGy}$ at 0.064 and $0.64 \mathrm{kBq}$ of ${ }^{211} \mathrm{At}$, respectively, would result in around $4 \%$ and $40 \%$ of single-hit cells in thyroid tissue (17). Because the lungs, liver, spleen, and kidney tissues received much lower absorbed doses at these activities, the fraction of cells traversed by $\alpha$-particles would be considerably lower than the nonhit population. Furthermore, differences in morphology and physiology of specialized cells imply differences in stress responses due to different homeostatic regulation and normal gene expression levels. Hence, without a separation of cell types, the analyzed transcriptional response is in fact a mixed response from differently responding cells. These aspects need to be regarded critically when comparing results from the investigated tissues: the differences in histologic structure, morphology, and cell density of tissues influence microdosimetric calculations, whereas the mixture of cell types influences the results of presumably cell-specific transcriptional responses - some of which would be direct responses to $\alpha$-particle hits and some might be nontargeted responses at low absorbed doses.

The increased ${ }^{211}$ At retention in the lungs is speculated to result in part from lymphocytes that are compartmentalized in pulmonary tissues (29). Immune activities in lymphocytes are modulated by thyroid hormones (30), and incorporation of ${ }^{211} \mathrm{At}$ into thyroid hormones could therefore increase the dose deposition in pulmonary tissues. Moreover, radioiodine uptake in the lungs has been linked to inflammatory responses in humans, suggesting a function of free iodine in basic immune activities (31). Putatively, ${ }^{211}$ At could be processed similarly by immune cells and thus retained. In this context, T-cell deficiency in BALB/c nude mice, compared with wild-type mice, might decrease ${ }^{211}$ At retention. The metallic character of ${ }^{211} \mathrm{At}-$ for example, conferred by the lower electronegativity compared with other halogens-is assumed to increase the retention in the liver and lungs and in the central nervous system, bone, and fatty tissues, where toxic metallic elements generally accumulate in the body.

The transcriptional response in all tissues was much higher at the 2 lowest ${ }^{211}$ At activities than at higher activities. In thyroid tissues, which were taken from the same mice as the tissues investigated in this study, a similar threshold phenomenon was also reported between 0.64 and $1.8 \mathrm{kBq}$ of ${ }^{211} \mathrm{At}$; however, this threshold corresponded to much higher absorbed doses (i.e., 50 and $500 \mathrm{mGy}$, respectively) (17). On the basis of the similar response in the thyroid (17) and the tissues investigated in this study, despite large differences in absorbed dose, it is hypothesized that this threshold phenomenon seen in tissues other than the thyroid is likely due to a thyroid-based systemic regulation rather than a tissue-based direct response to radiation exposure. Theoretically, 3 basic stages would be distinguishable for the tissues in dependence of increasing
${ }^{211}$ At activity: first, a dominance of thyroid-induced response; second, a concomitance of thyroid-induced and radiation-induced response; and third, a dominance of radiation-induced response. A thyroid-induced response is likely based on thyroid hormones, which would be dysregulated because of thyroid damage at sufficiently high absorbed doses from ${ }^{211}$ At. This hypothesis is supported by previously presented findings: the biological process of thyroid hormone generation was affected at 1.4 and 11 Gy absorbed dose (17). Moreover, Ttr (encoding for the carrier of the thyroid hormone thyroxine) was downregulated at all absorbed doses, and Slc5a8 (Solute carrier family 5 , member 8) was downregulated at $1.4 \mathrm{~Gy}$ but upregulated at all other absorbed doses (17). Potentially, there could also be additional radiation-induced signaling factors originating from the affected thyroid on ionizing radiation exposure, which could mediate a nontargeted or systemic bystander response in vivo.

The low response in previously proposed radiationassociated genes, which was understood as a limitation between in vitro and in vivo studies impeding a direct translation of results between the 2 systems, was also reported for the thyroid (17). In this study, various genes may suggest a response threshold in some tissues between 0.64 and $1.8 \mathrm{kBq}$ of ${ }^{211} \mathrm{At}$, but the incidence was comparatively low considering all investigated genes associated with radiation responses. Furthermore, the regulation of these genes was generally at a low response level. Pcna was the only gene regulated for at least 1 absorbed dose in the tissues. Interestingly, in the kidney cortex and medulla, liver, and lungs, Pcna was upregulated at 0.064 or $0.64 \mathrm{kBq}$ of ${ }^{211} \mathrm{At}$. In the spleen, however, Pcna was downregulated at $1.8-42 \mathrm{kBq}$ of ${ }^{211} \mathrm{At}$, suggesting threshold behavior. The role of PCNA is versatile because of numerous PCNA-interacting proteins involved in various cellular pathways (32). Thus, cellular effects cannot be derived from Pcna transcription patterns without its regulatory context. Amy 2 showed an exceptionally high and nonmonotonous upregulation in the spleen above the observed threshold. This nonmonotonous absorbed dose-dependence shown in vivo for ${ }^{211}$ At exposure needs to be regarded critically when further evaluating Amy2 as a potential biomarker for ionizing radiation exposure.

The regulation of associated biological processes reflected the threshold phenomenon between 0.64 and 1.8 $\mathrm{kBq}$ of ${ }^{211} \mathrm{At}$, which was an expected result taking into consideration the strong decrease in up- and downregulation of transcripts between these administered activities. The categorization of biological processes after higherlevel cellular function revealed comprehensive profiles for each tissue. Radiation metabolomics has shown the feasibility of metabolites as biomarkers for ionizing radiation exposure, suggesting that regulation profiles of metabolic processes have indicative potential. Recently, Johnson et al. (33) identified several urinary biomarkers in nonhuman primates, among which taurine and xanthine had been identified 
previously in the mouse $(34,35)$. In fact, responses in genes associated with taurine and xanthine metabolization were regulated in some instances (data not shown). Interestingly, the spleen showed the most distinct regulation profile when compared with the other tissues, with all categories displaying the threshold phenomenon except for metabolism and organismic regulation. The spleen also featured the highest number of subcategories $(8 / 31)$ that were not regulated at any exposure in contrast to the other tissues $(4 / 31$ or $5 / 31$ ), suggesting a more specific and less diverse overall response. These findings could be related to the high amount of blood cells in spleen tissue. The lungs also displayed the regulatory threshold behavior for various subcategories, despite much higher absorbed doses, indicating that these observed responses cannot be solely related to absorbed dose. The observed tissue-specific regulatory patterns pose an interesting aspect for long-term studies and risk estimations. However, from this type of data it could not be concluded whether regulations of subcategories in a tissue were a parallel response to an initial stimulus or whether the regulation of a subcategory was a response to regulatory changes in another subcategory.

In general, the identification of tissue-specific responses would be in agreement with Zhao et al. (36), who discovered strikingly different gene expression patterns in normal mouse kidney and brain tissue on ionizing radiation exposure. This finding challenged the assumption that radiationinduced changes in normal tissue are preprogrammed genetic changes that are not influenced by tissue-specific origin (36). However, Zhao et al. used a different radiation quality and exposure modality-that is, external whole-body irradiation with a single dose of $10 \mathrm{~Gy}$ of ${ }^{137} \mathrm{Cs} \gamma$-rays-which impeded a direct comparison with the results presented here. Furthermore, in this study, the absorbed doses were mainly in the low-dose range, and it is hypothesized that overlaying organismic regulations from the thyroid influenced the regulation profiles as well.

Differential gene regulation across all absorbed doses suggested potential in vivo biomarkers for the kidney cortex, lungs, and spleen. In the kidney cortex, the extent of downregulation of 2310057J18Rik (encoding for a protein of unknown function) showed a minor but monotonous decrease, with an increased absorbed dose from $2.4 \mathrm{mGy}$ to higher absorbed doses. In the lungs, the extent of suppression of Hspl05 (Heat shock protein $105 \mathrm{kDa}$ ) decreased with increased absorbed dose. However, this response was not sensitive between a 16- and a 350-mGy absorbed dose. In the liver, the upregulation of Rgs16 (regulator of G-protein signaling 16) increased with increased absorbed dose from $2.0 \mathrm{mGy}$ to higher absorbed doses. These candidate genes should be tested for use as biomarkers in further studies investigating, for example, the regulatory response over time, a wider range or higher resolution of intravenously administered ${ }^{211} \mathrm{At}$ activities, different radiation qualities, and similarities and differences in responses between different mouse strains.

\section{CONCLUSION}

This study showed a more pronounced transcriptional response at lower absorbed doses than at higher absorbed doses from intravenously administered ${ }^{211} \mathrm{At}$ in the kidney cortex and medulla, liver, lungs, and spleen of BALB/c nude mice. Threshold behavior in terms of a strong decrease in regulatory intensity was observed for the total number of regulated transcripts as the injected activity increased from 0.64 to $1.8 \mathrm{kBq}$ of ${ }^{211} \mathrm{At}$. A similar response was also reported for the thyroid in a preceding study (17), although the investigated abdominal organs and the lungs had received much lower levels of absorbed dose than the thyroid. The threshold behavior was also reflected by the regulation of biological processes when categorized after higher-level cellular function, revealing tissue-specific regulatory patterns. The regulation of previously proposed radiation-associated genes was on a low level in this in vivo study but indicated tissue-specific responses. In all tissues, few genes were regulated at all absorbed doses, generally showing no absorbed dose dependence.

The pronounced regulatory change observed between 0.64 and $1.8 \mathrm{kBq}$ of ${ }^{211} \mathrm{At}$ may partly be interpreted as a response to thyroid-based signaling and not as exclusively based on radiation-induced responses in each tissue itself. This observation indicates the significance of physiology when investigating radiation-induced effects in vivo. In this regard, further questions need to be addressed. The sensitivity of organs for radiation-induced thyroid dysregulation and damage is an important aspect. In addition, the boundary conditions for induction of this response - that is, radiation quality, absorbed dose, dose rate, and time of on-setneed to be studied in further detail.

\section{DISCLOSURE}

The costs of publication of this article were defrayed in part by the payment of page charges. Therefore, and solely to indicate this fact, this article is hereby marked "advertisement" in accordance with 18 USC section 1734. This study was supported by grants from the European Commission FP7 Collaborative Project TARCC HEALTH-F2-2007201962, the Swedish Research Council (grant 21073), the Swedish Cancer Society (grant 3427), BioCARE-a National Strategic Research Program at the University of Gothenburg, the Swedish Radiation Safety Authority, the King Gustav V Jubilee Clinic Cancer Research Foundation, the Sahlgrenska University Hospital Research Funds, and the Assar Gabrielsson Cancer Research Foundation. The work was performed within the EC COST Action BM0607. No other potential conflict of interest relevant to this article was reported.

\section{ACKNOWLEDGMENTS}

We thank Lilian Karlsson and Ann Wikström for their valuable technical assistance and Szilárd Nemes for the helpful discussions on statistical issues. We also thank 
Dr. Holger Jensen at Rigshospitalet, Copenhagen, Denmark, and Drs. Sture Lindgren, Sofia Frost, and Tom Bäck for their assistance with ${ }^{211} \mathrm{At}$.

\section{REFERENCES}

1. Corson DE, MacKenzie KR, Segrè E. Artificially radioactive element 85. Phys Rev. 1940;58:672-678

2. Zalutsky MR, Bigner DD. Radioimmunotherapy with a-particle emitting radioimmunoconjugates. Acta Oncol. 1996;35:373-379.

3. Harrison A. The application of ${ }^{211} \mathrm{At}$ in experimental tumor therapy. Radiochem Acta. 1989;47:157-161.

4. Larsen RH, Akabani G, Welsh P, Zalutsky MR. The cytotoxicity and microdosimetry of astatine-211-labeled chimeric monoclonal antibodies in human glioma and melanoma cells in vitro. Radiat Res. 1998;149:155-162.

5. Andersson H, Cederkrantz E, Bäck T, et al. Intraperitoneal alpha-particle radioimmunotherapy of ovarian cancer patients: pharmacokinetics and dosimetry of ${ }^{211}$ At-MX35 F(ab')2-a phase I study. J Nucl Med. 2009;50:1153-1160.

6. Andersson H, Lindegren S, Back T, Jacobsson L, Leser G, Horvath G. The curative and palliative potential of the monoclonal antibody MOv18 labelled with ${ }^{211}$ At in nude mice with intraperitoneally growing ovarian cancer xenografts-a long-term study. Acta Oncol. 2000;39:741-745.

7. Hamilton JG, Durbin PW, Parrott M. The accumulation and destructive action of astatine 211 (eka-iodine) in the thyroid gland of rats and monkeys. J Clin Endocrinol Metab. 1954;14:1161-1178.

8. Hamilton JG, Durbin PW, Parrott MW. Accumulation of astatine211 by thyroid gland in man. Proc Soc Exp Biol Med. 1954;86:366-369.

9. Brown I. Astatine-211: its possible applications in cancer therapy. Int J Rad Appl Instrum [A]. 1986;37:789-798.

10. Lindencrona U, Nilsson M, Forssell-Aronsson E. Similarities and differences between free ${ }^{211} \mathrm{At}$ and ${ }^{125} \mathrm{I}$ - transport in porcine thyroid epithelial cells cultured in bicameral chambers. Nucl Med Biol. 2001;28:41-50.

11. Lundh C, Lindencrona U, Postgård $\mathrm{P}$, Carlsson $\mathrm{T}$, Nilsson $\mathrm{M}$, ForssellAronsson E. Radiation-induced thyroid stunning: differential effects of ${ }^{123} \mathrm{I}$, ${ }^{131} \mathrm{I},{ }^{99 \mathrm{~m}} \mathrm{Tc}$, and ${ }^{211} \mathrm{At}$ on iodide transport and NIS mRNA expression in cultured thyroid cells. J Nucl Med. 2009;50:1161-1167.

12. Lundh C, Lindencrona U, Schmitt A, Nilsson M, Forssell-Aronsson E. Biodistribution of free ${ }^{211} \mathrm{At}$ and ${ }^{125} \mathrm{I}$ in nude mice bearing tumors derived from anaplastic thyroid carcinoma cell lines. Cancer Biother Radiopharm. 2006;21: 591-600.

13. Kruse JJ, te Poele JA, Velds A, et al. Identification of differentially expressed genes in mouse kidney after irradiation using microarray analysis. Radiat Res. 2004;161:28-38.

14. Taki K, Wang B, Nakajima T, et al. Microarray analysis of differentially expressed genes in the kidneys and testes of mice after long-term irradiation with low-dose-rate gamma-rays. J Radiat Res (Tokyo). 2009;50:241-252.

15. Snyder AR, Morgan WF. Gene expression profiling after irradiation: clues to understanding acute and persistent responses? Cancer Metastasis Rev. 2004;23: 259-268.

16. Chaudhry MA. Biomarkers for human radiation exposure. J Biomed Sci. 2008; 15:557-563.

17. Rudqvist N, Parris TZ, Schüler E, Helou K, Forssell-Aronsson E. Transcriptional response of $\mathrm{BALB} / \mathrm{c}$ mouse thyroids following in vivo astatine-211 exposure reveals distinct gene expression profiles. EJNMMI Res. June 14, 2012 [Epub ahead of print].

18. Bentzen SM. Preventing or reducing late side effects of radiation therapy: radiobiology meets molecular pathology. Nat Rev Cancer. 2006;6:702-713.

19. Prise KM, Schettino G, Folkard M, Held KD. New insights on cell death from radiation exposure. Lancet Oncol. 2005;6:520-528.

20. Rubin P, Johnston CJ, Williams JP, McDonald S, Finkelstein JN. A perpetual cascade of cytokines postirradiation leads to pulmonary fibrosis. Int $J$ Radiat Oncol Biol Phys. 1995;33:99-109.

21. Lindegren S, Back T, Jensen HJ. Dry-distillation of astatine-211 from irradiated bismuth targets: a time-saving procedure with high recovery yields. Appl Radiat Isot. 2001;55:157-160.

22. Bolch WE, Eckerman KF, Sgouros G, Thomas SR. MIRD pamphlet no. 21: a generalized schema for radiopharmaceutical dosimetry-standardization of nomenclature. J Nucl Med. 2009;50:477-484.

23. Garg PK, Harrison CL, Zalutsky MR. Comparative tissue distribution in mice of the alpha-emitter ${ }^{211}$ At and ${ }^{131} \mathrm{I}$ as labels of a monoclonal antibody and $\mathrm{F}(\mathrm{ab}$ ')2 fragment. Cancer Res. 1990;50:3514-3520.

24. International Commission on Radiation Units and Measurements. Stopping Powers and Ranges for Protons and Alpha Particles. Bethesda, MD: International Commission on Radiation Units and Measurements; 1993.

25. Parris TZ, Danielsson A, Nemes S, et al. Clinical implications of gene dosage and gene expression patterns in diploid breast carcinoma. Clin Cancer Res. 2010;16:3860-3874.

26. Benjamini YHY. Controlling the false discovery rate: a practical and powerful approach to multiple testing. J R Stat Soc, B. 1995;57:289-300.

27. Ashburner M, Ball CA, Blake JA, et al. Gene ontology: tool for the unification of biology. The Gene Ontology Consortium. Nat Genet. 2000;25:25-29.

28. Josefsson A, Forssell-Aronsson E. Microdosimetric analysis of ${ }^{211}$ At in thyroid models for man, rat and mouse. EJNMMI Res. June 9, 2012 [Epub ahead of print].

29. Pabst R, Tschernig T. Lymphocytes in the lung: an often neglected cellnumbers, characterization and compartmentalization. Anat Embryol (Berl). 1995; 192:293-299.

30. De Vito P, Incerpi S, Pedersen JZ, Luly P, Davis FB, Davis PJ. Thyroid hormones as modulators of immune activities at the cellular level. Thyroid. 2011;21:879890.

31. Triggiani V, Moschetta M, Giagulli VA, Licchelli B, Guastamacchia E. Diffuse Iodine-131 lung uptake in bronchiectasis: a potential pitfall in the follow up of differentiated thyroid carcinoma. Thyroid. 2012;22:9-16.

32. Maga G, Hubscher U. Proliferating cell nuclear antigen (PCNA): a dancer with many partners. J Cell Sci. 2003;116:3051-3060.

33. Johnson CH, Patterson AD, Krausz KW, et al. Radiation metabolomics. 5. Identification of urinary biomarkers of ionizing radiation exposure in nonhuman primates by mass spectrometry-based metabolomics. Radiat Res. 2012;178: 328-340.

34. Tyburski JB, Patterson AD, Krausz KW, et al. Radiation metabolomics. 1. Identification of minimally invasive urine biomarkers for gamma-radiation exposure in mice. Radiat Res. 2008;170:1-14.

35. Tyburski JB, Patterson AD, Krausz KW, et al. Radiation metabolomics. 2. Doseand time-dependent urinary excretion of deaminated purines and pyrimidines after sublethal gamma-radiation exposure in mice. Radiat Res. 2009;172:42-57.

36. Zhao W, Chuang EY, Mishra M, et al. Distinct effects of ionizing radiation on in vivo murine kidney and brain normal tissue gene expression. Clin Cancer Res. 2006;12:3823-3830. 